LAW RENCE LIVERMORE N A TIO N A L LABORATORY

Regulation of Yersina pestis Virulence by Al-2 Mediated Quorum Sensing

B. Segelke, S. Hok, V. Lao, M. Corzett, E. Garcia

April 9, 2010 
This document was prepared as an account of work sponsored by an agency of the United States government. Neither the United States government nor Lawrence Livermore National Security, LLC, nor any of their employees makes any warranty, expressed or implied, or assumes any legal liability or responsibility for the accuracy, completeness, or usefulness of any information, apparatus, product, or process disclosed, or represents that its use would not infringe privately owned rights. Reference herein to any specific commercial product, process, or service by trade name, trademark, manufacturer, or otherwise does not necessarily constitute or imply its endorsement, recommendation, or favoring by the United States government or Lawrence Livermore National Security, LLC. The views and opinions of authors expressed herein do not necessarily state or reflect those of the United States government or Lawrence Livermore National Security, LLC, and shall not be used for advertising or product endorsement purposes.

This work performed under the auspices of the U.S. Department of Energy by Lawrence Livermore National Laboratory under Contract DE-AC52-07NA27344. 


\title{
Regulation of Yersina pestis Virulence by AI-2 Mediated Quorum Sensing
}

Brent Segelke, Saphon Hok, Victoria Lao, Michele Corzett, and Emilio Garcia

\author{
Physical and Life Sciences \\ Biology and Biotechnology Division \\ Lawrence Livermore National Laboratory \\ LDRD-LW08 Final Report
}




\section{Motivation}

The proposed research was motivated by an interest in understanding $Y$. pestis virulence mechanisms and bacteria cell-cell communication. It is expected that a greater understanding of virulence mechanisms will ultimately lead to biothreat countermeasures and novel therapeutics. $Y$. pestis is the etiological agent of plague, the most devastating disease in human history. $Y$. pestis infection has a high mortality rate and a short incubation before mortality.[1] There is no widely available and effective vaccine for $Y$. pestis and multi-drug resistant strains are emerging. $Y$. pestis is a recognized biothreat agent based on the wide distribution of the bacteria in research laboratories around the world and on the knowledge that methods exist to produce and aerosolize large amounts of bacteria.[2]

We hypothesized that cell-cell communication via signaling molecules, or quorum sensing, by $Y$. pestis is important for the regulation of virulence factor gene expression during host invasion, though a causative link had never been established. Quorum sensing is a mode of intercellular communication which enables orchestration of gene expression for many bacteria as a function of population density and available evidence suggests there may be a link between quorum sensing and regulation of $Y$. pesits virulence. Several pathogenic bacteria have been shown to regulate expression of virulence factor genes, including genes encoding type III secretion, via quorum sensing.[3-5] The $Y$. pestis genome encodes several cell-cell signaling pathways and the interaction of at least three of these are thought to be involved in one or more modes of host invasion.[2] Furthermore, $Y$. pestis gene expression array studies carried out at LLNL have established a correlation between expression of known virulence factors and genes involved in processing of the AI-2 quorum sensing signal. [6]

\section{Scope}

This was a basic research project that was intended to provide new insights into bacterial intercellular communication and how it is used to regulate virulence in $Y$. pestis. It is known that many bacteria use intercellular signaling molecules to orchestrate gene expression and cellular function. A fair amount is known about production and uptake of signaling molecules, but very little is known about how intercellular signaling regulates other pathways.[7] Although several studies demonstrate that intercellular signaling plays a role in regulating virulence in other pathogens, the link between signaling and regulation of virulence has not been established. Very little work had been done directly with $Y$. pestis intercellular signaling apart from the work carried out at LLNL. The research we proposed was intended to both establish a causative link between AI-2 intercellular signaling and regulation of virulence in $Y$. pestis and elucidate the fate of the AI-2 signaling molecule after it is taken up and processed by $Y$. pestis. Elucidating the fate of AI-2 was expected to lead directly to the understanding of how AI-2 signal processing regulates other pathways as well as provide new insights in this direction.

\section{Research plan and expected results Background}

Bacteria are able to communicate via chemical signals called autoinducers. Bacteria cells respond to autoinducer levels in a process called quorum sensing.[7] Quorum sensing is used to sense population density changes and to orchestrate cellular function, including regulation of virulence in some pathogens, as a community (figure 1). A fair amount of work has been done elucidating pathways for the production and uptake of autoinducer molecules, but little is known about how sensing of autoinducers regulates other pathways.[7] Efforts at LLNL and elsewhere 
have identified a number of the autoinducer 1 (AI-1) class of molecules produced by $Y$. pestis, but very little is know about the function of the AI-2 system in Y.pestis.[8] The Y. pestis genome

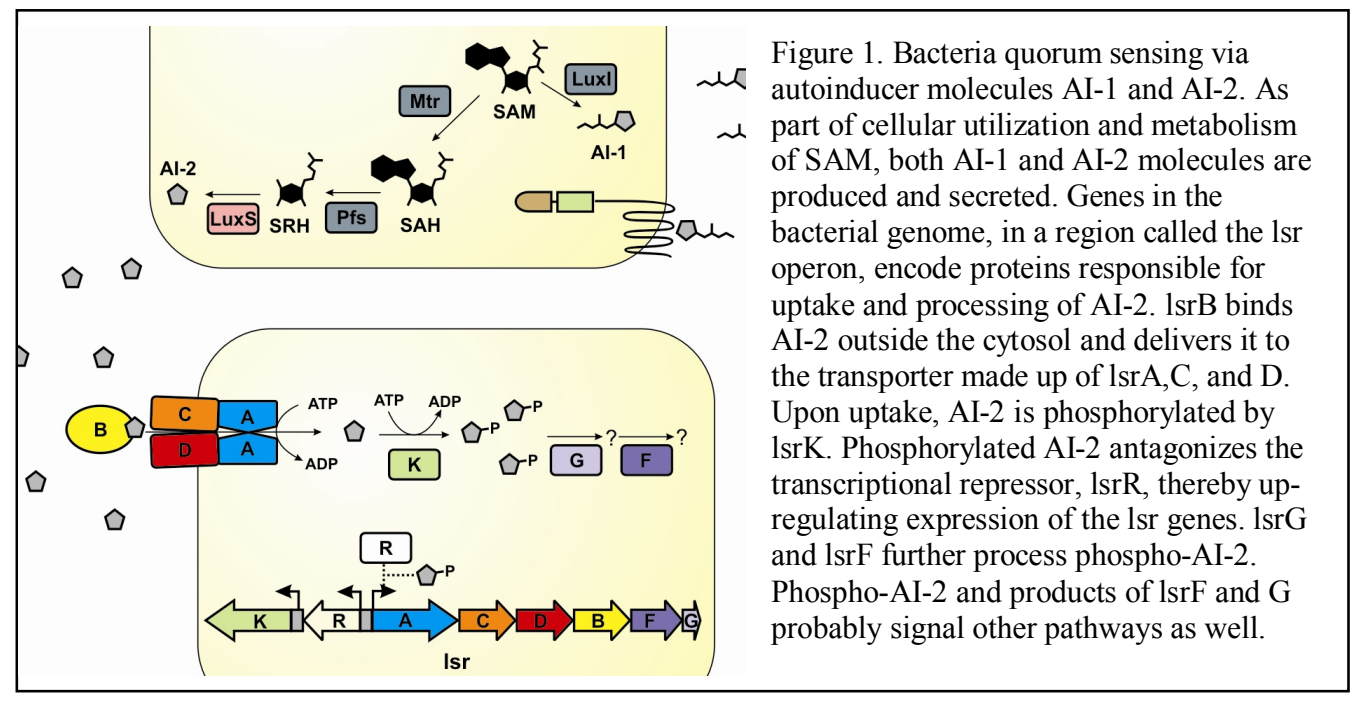

encodes the lsr operon (see figure 1) and we previously demonstrated that $Y$. pestis produces and takes up AI-2 activity. We also previously determined the X-ray crystal structure of lsrG from $Y$. pestis.

We originally proposed the following specific aims

We proposed to establish a causative link between the uptake and processing of AI-2 and its regulation of virulence factor genes in $Y$. pestis and to elucidate AI-2 processing pathways. We proposed to do so by pursuing three complementary lines of investigation. First, proposed to observe the effects of specific engineered genetic mutation, which alter different aspects of AI-2 uptake and processing, on expression of known virulence factor genes. Second, we proposed to track the fate of AI-2 as it is taken up and processed by $Y$. petsis using radiocarbon labeled AI-2 and to identify AI-2 metabolites by mass spectroscopy and accelerator mass spectroscopy. This is a novel approach to studying cell-cell signaling that leverages unique LLNL capabilities. Using AMS in this way is obviously generalizable, and likely a very powerful way to examine a pathogens interactions with its environment. Finally, proposed to elucidate the substrates and products of the enzymes encoded in the lsr operon, which encodes the genes for AI-2 uptake and processing (figure 1). We proposed to carry out the following tasks:

1. Engineer genetic mutant strains, or knockouts, of $Y$. pestis deficient in individual genes required for AI-2 production, uptake, and processing (luxS, lsrA, 1srK, lsrF, and lsrG)

2. Monitor the impact of specific knockouts on expression of known virulence factor genes (yersinia outer proteins, yops, and low calcium response genes) by RT-PCR

3. Synthesize AI-2 and radiocarbon labeling AI-2

4. Isolate radio carbon label containing metabolites generated in the cytosol of $Y$. pestis following uptake of exogenous radiocarbon labeled AI-2

5. Identify AI-2 metabolites by mass spectroscopy

6. Isolate gene products (proteins) known to be involved in AI-2 uptake and processing 7. Identify substrates and products (metabolites) in the known AI-2 processing pathway

Engineering genetic knockouts in Y. pestis KIM D27:

We attempted to generate knockout mutants using the Datsenko and Wanner one-step inactivation method of chromosomal bacterial genes using PCR products.[9] This method works by homologous recombination where a recombinase and an engineered linear 
segment of DNA carrying recombinase sites are introduced into the bacteria to be genetically modified. The DNA segment is designed such that the recombinase replaces the target gene with the engineered DNA segment. We have experience using this method to knock out genes in $Y$. pestis. Regulatory restriction on this work turned out to be a much more significant hurdle than we had anticipated. Methods we use confer transient antibiotic resistance to the target organism is now highly restricsted for virulent organisms such as $Y$. pestis. Despite using avirulent strain of Y. pestis (KIM D27) our IBC committee required that we work under the restrictions applied to fully virulent $Y$. pestis. It took us approximately 2 years to overcome the restrictions and develop a genetic system that works in the avirulent strains of $Y$. pestis we used. We were able to generate one genetic knockout at the end of the project and have yet to test the phenotype of the mutant.

Monitoring expression of virulence factor genes

Since we were not able to produce the genetic knockouts until the end of the project we were not able to carry out this part of the proposed work.

Chemical synthesis of AI-2

We completed the chemical synthesis of AI-2 and $\left[{ }^{14} \mathrm{C}\right] \mathrm{AI}-2$.

Tracking AI-2 metabolites with AMS

Delays in generating genetic knockouts significantly delayed the start of these experiments. We are now poised to initiate these experiments and we are pursuing support to continue this work.

Isolating proteins involved in AI-2 uptake and processing

We were able to isolate and characterize $1 \mathrm{srB}, 1 \mathrm{srK}, 1 \mathrm{srF}, 1 \mathrm{srG}$ and some independently folding subdomains of ypo0405. We were not able to isolate an active form of 1srR and we did not attempt to isolate lsrA, B, and C, since these are membrane associated proteins.

Elucidating metabolites of AI-2 processing

We were able to generate products of AI-2 processing, but have yet to fully characterize them.

Additional work

In addition to the originally proposed work we collaborated with computational biology to predict protein-protein interactions amongst the proteins encoded in the lsr operon and worked to experimentally validate the predicted interactions. We also obtained the crystal structure of lsrF and have crystals of AI-2 bound to lsrB. 
References:

1. Zhou D, Han Y, Yang R, (2006), Molecular and physiological insights into plague transmission, virulence and etiology, Microbes and Infection 8: 273-284

2. NIAID Biodefense Research Agenda for CDC Category A Agents, U. S. DEPARTMENT OF HEALTH AND HUMAN SERVICES, National Institutes of Health, National Institute of Allergy and Infectious Diseases 2002.

3. Laasik E, Andresen L, Mae A, (2006), Type II quorum sensing regulates virulence in Erwinia carotovora ssp. carotovora, FEMS Miocrobiology Letters 258: 227-34

4. Yahr1 TL, Wolfgang MC, (2006), Transcriptional regulation of the Pseudomonas aeruginosa type III secretion system, Molecular Microbiology 62: 631-40

5. Krin E, Chakroun N, Turlin E, Givaudan A, Gaboriau F, Bonne I, Rousselle J-C, Frangeul, Lacroix C, Hullo M-F, Marisa L, Danchin A, Derzelle S, (2006), Pleiotropic Role of Quorum Sensing Autoinducer 2 in Photorhabdus luminescens, Applied and Environmental Microbiology October 2006: 6439-51

6. Motin VL, Georgescu AM, Fitch JP, Gu PP, Nelson DO, Mabery SL, Garnham JB, Sokhansanj BA, Ott LL, Coleman MA, Elliott JM, Kegelmeyer LM, Wyrobek AJ, Slezak TR, Brubaker RR, Garcia E, (2004), Temporal global changes in gene expression during temperature transition in Yersinia pestis, Journal of Bacteriology 186: 6298-305

7. Camilli A, Bassler BL, (2006), Bacterial small-molecule signaling pathways, Science 311: 1113-6

8. Chen Z, Li B, Zhang J, Qin L, Zhou D, Han Y, Du Z, Guo Z, Song Y, Yang R , (2006), Quorum sensing affects virulence-associated proteins F1, LcrV, KatY and pH6 etc. of Yersinia pestis as revealed by protein microarray-based antibody profiling, Microbes and Infection 8: 2501-8

9. Datsenko KA, Wanner BL, (2000), One-step inactivation of chromosomal genes in Escherichia coli K-12 using PCR products, The Proceedings of the National Academy of Science. USA 97:6640-5

10. Semmelhack MF, Campagna SR, Federle MJ, Bassler BL, (2005), An expeditious synthesis of DPD and boron binding studies, Organic Letters 7: 569-72

11. De Keersmaecker SC, Varszegi C, van Boxel N, Habel LW, Metzger K, Daniels R, Marchal K, De Vos D, Vanderleyden J, (2005), Chemical synthesis of (S)-4,5-dihydroxy-2,3pentanedione, a bacterial signal molecule precursor, and validation of its activity in Salmonella typhimurium, The Journal of Biological Chemistry 280: 19563-8 